Results 26/103 (25.2\%) STRA children were classified with PAL. There were no differences in the demographic characteristics between the groups. Fewer children with PAL had a previous Paediatric Intensive Care Unit admission $(21.7 \%$ versus 47.1\%); there were no other differences in asthma control. Children with PAL had a higher number of submucosal eosinophils $(p=0.021)$ in endobronchial biopsies before triamcinolone, but there were no differences in airway luminal inflammation in BAL. However, there was a trend towards lower sputum eosinophils post, but not pre, triamcinolone in children with PAL $(0.65 \% \quad(0-17)$ versus $2.5 \% \quad(0-42.8)$, $\mathrm{p}=0.054)$. There were no differences in blood eosinophils or FENO levels. All children classified as PAL post triamcinolone continued to have reduced $\mathrm{FEV}_{1}$ (post bronchodilator $\mathrm{z}$ score <-1.96) in the following 12 months.

Conclusion PAL is relatively common in paediatric STRA even when a very stringent definition is used. Mucosal eosinophilic inflammation is associated with PAL and may represent a therapeutic target. Further work is needed to elucidate underlying mechanisms.

\section{S73 COUGH FREQUENCY AND DIURNAL PATTERNS IN CHILDREN WITH ASTHMA}

D Elghamoudi, K McGuinness, J Smith, C Murray. Division of Infection, Immunity and Respiratory Medicine, Faculty of Biology, Medicine and Health, Manchester Academic Health Sciences Centre, University of Manchester and University Hospital of South Manchester NHS Foundation Trust, Manchester, UK

\subsection{6/thoraxjnl-2017-210983.79}

Background Asthma is one of the commonest childhood diseases. Although cough is considered a key symptom of asthma, little is known about the cough patterns in asthmatic children. Previously, cough patterns in asthma have only been studied subjectively, in terms of frequency and diurnal variation, and it has been reported that asthmatics cough more at night, specifically from midnight until early morning. However, there is little objective data to confirm this. Using an objective cough monitoring system we investigated cough frequency and diurnal patterns of cough over 24 hours in asthmatic children.

Methods Children (age 2-17 years) with a diagnosis of Asthma were asked to wear the VitaloJAK cough monitor for a maximum of 24 hours on two occasions - when symptoms were stable and during an exacerbation. All 24 hour recordings were processed through compression software and coughs counted by listening to the resulting files; the 24 hour cough number were reported.

Results 26 stable asthmatic children (17 male; median age 11.9 years) completed 24 hour recordings when stable; 12 repeated recordings during an exacerbation. During the stable period median total cough counts during awake-hours, sleephours and 24 hour periods were 69.5 (range 3-395), 0 (range 0-151) and 71 (range 4-432) respectively. Coughs occurred mostly during the awake time (86\%), peaking at 08:00 and 19:00 hours, and rarely occurred during the night when subjects were sleeping (14\%). During exacerbations the median total cough counts during awake-hours, sleep-hours and 24 hours were 183 (range 0-632), 18.5 (range 0-128) and 262 (range 10-645), which were significantly higher than during stable recordings $(\mathrm{p}<0.05)$. The incidences of day-time cough were similar to that of stable asthmatic recordings $(80 \%)$ and the incidences of night-time cough remained low (10\%). However, during an exacerbation, the peak time of coughing was at 11:00 and 17:00-19:00 hours. The distribution of cough for 12 asthmatic children when stable and during exacerbations is shown in figure 1.

Conclusions Objective monitoring shows that cough frequency in children is greater during the day and was reduced during

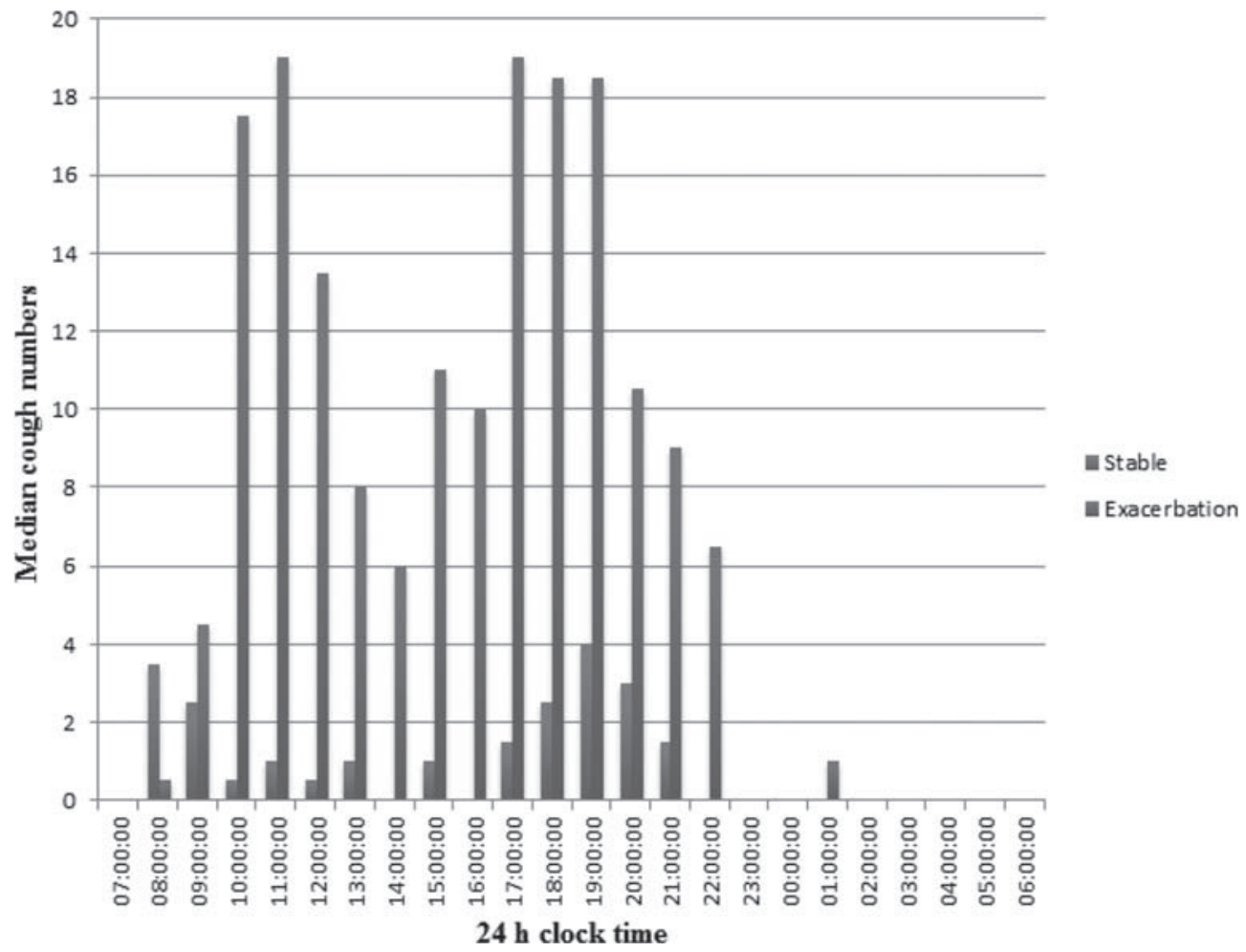

Abstract S73 Figure 1 Cough frequency distribution during exacerbations and stable asthma for 12 asthmatic children. 
sleep in both stable and exacerbating asthma. Cough frequency increases during the morning and late afternoon.

\section{Mechanistic insights into interstitial lung disease}

\section{S74 ENDOPLASMIC STRESS IS ASSOCIATED WITH FIBROSIS IN INTERSTITIAL LUNG DISEASE}

${ }^{1} \mathrm{H}$ Parfrey, ${ }^{2} \mathrm{E}$ Moseley, ${ }^{2} \mathrm{~B}$ Beardsley, ${ }^{2} \mathrm{~J}$ Knight, ${ }^{3} \mathrm{SJ}$ Marciniak, ${ }^{2} \mathrm{D}$ Rassl. ${ }^{1}$ Papworth Hospital NHSFT, Cambridge, UK; ${ }^{2}$ Department of Pathology, Papworth Hospital NHSFT, Cambridge, UK; ${ }^{3}$ Department of Medicine, CIMR, University of Cambridge, Cambridge, UK

10.1136/thoraxinl-2017-210983.80

Interstitial lung diseases (ILD) are a heterogeneous group characterised by variable amounts of inflammation and fibrosis. However, the development of pulmonary fibrosis is associated with a poorer prognosis. Although distinct histological features differentiate between the ILDs, it is unknown if there are shared pathogenic mechanisms involved in the development of fibrosis. Endoplasmic reticulum (ER) stress has been implicated in the pathogenesis of familial and sporadic idiopathic pulmonary fibrosis (IPF). In response to ER stress, cells trigger the unfolded protein response (UPR) and upregulate chaperones, such as BiP, and the phosphatase GADD34, which can regulate epithelial to mesenchyme transition, cell proliferation, apoptosis and cell survival.

Aims We hypothesise that ER stress may be involved in the pathogenesis of fibrosis in all interstitial lung diseases.

Methods Paraffin embedded sections, obtained from video assisted thoracoscopic diagnostic lung biopsies, from 8 patients with familial pulmonary fibrosis, 11 sporadic idiopathic pulmonary fibrosis (IPF), 12 non-specific interstitial pneumonia (NSIP) and 10 hypersensitivity pneumonitis (HP) were evaluated for $\mathrm{BiP}$ and GADD34 by immunohistochemistry. Using light microscopy, 6 high power fields were scored for fibrosis, inflammation, BiP and GADD34 using semi-quantitative analysis by 2 blinded, independent investigators. Data were analysed by linear regression using Prism software.

Results Of the 41 biopsy samples analysed, 20 (49\%) were non-smokers and 18 (44\%) were male. BiP and GADD34 were localised to reactive type II pneumocytes and columnar epithelium within areas of fibrosis. GADD34 was also evident in the endothelium. No staining was detected within fibroblasts or fibroblastic foci. Epithelial GADD34 correlated with extent of fibrosis in familial pulmonary fibrosis $\left(\mathrm{r}^{2}=0.72 \mathrm{p}<0.001\right), \quad$ IPF $\quad\left(\mathrm{r}^{2}=0.51 \mathrm{p}<0.0001\right)$ and NSIP $\left(r^{2}=0.46 \mathrm{p}<0.0001\right)$. In contrast, BiP was associated with fibrosis in IPF $\left(r^{2}=0.49 \mathrm{p}<0.0001\right)$ and HP $\left(\mathrm{r}^{2}=0.59 \mathrm{p}<0.0001\right)$. There was no association with inflammation.

Conclusion These data show that ER stress and the UPR are associated with fibrotic ILDs. Hence targeting ER stress may be a novel therapeutic option for pulmonary fibrosis. Work is on going to identify a peripheral biomarker signature for ER stress.

\section{S75 CYCLICAL STRETCH INDUCES G $\alpha$ Q/11 MEDIATED TGF $\beta$ ACTIVATION IN LUNG FIBROBLASTS}

${ }^{1} \mathrm{AT}$ Goodwin, ${ }^{1} \mathrm{AL}$ Tatler, ${ }^{2} \mathrm{~S}$ Offermanns, ${ }^{1} \mathrm{G}$ Jenkins. ${ }^{1}$ University of Nottingham, Nottingham, UK; ${ }^{2}$ Max Planck Institute for Heart and Lung Research, Bad Nauheim, Germany

\subsection{6/thoraxjnl-2017-210983.81}

Introduction Idiopathic pulmonary fibrosis (IPF) is an incurable and progressive fibrotic condition of the lung with a poor prognosis. Activation of the profibrotic cytokine transforming growth factor- $\beta$ (TGF $\beta$ ) is central to the pathogenesis of IPF. Mechanical signals are emerging as key mediators of TGF $\beta$ activation, and cyclical stretch is a ubiquitous stimulus in the lungs. Stretch of fibrotic lung induces TGF $\beta$ activation, and alveolar epithelial $G_{\alpha q / 11}$ signalling is important in stretchmediated TGF $\beta$ activation. However, the role of $G_{\alpha q / 11}$ signalling in stretch-mediated TGF $\beta$ activation by lung fibroblasts is unclear.

Methods Murine embryonic fibroblasts (MEFs) and human lung fibroblasts (HLFs) were subject to cyclical stretch using the Flexcell system, and stretch regimens designed to mimic tidal breathing in the relevant organism $(15 \%$ elongation unless otherwise stated, frequency: $1 \mathrm{~Hz}$ in MEFs, $0.3 \mathrm{~Hz}$ in HLFs). Phosphorylated Smad2 (pSmad2), total Smad2, and alpha smooth muscle actin ( $\alpha \mathrm{SMA}$ ) protein expression were assessed by western blot. TGF $\beta$ activation was determined using the ratio of $\mathrm{pSmad} 2$ to total $\mathrm{Smad} 2$ on densitometry. Comparisons of stretch-mediated TGF $\beta$ activation were made between wild-type (WT), $G_{\alpha 12 / 13^{-}}$, and $G_{\alpha q / 11^{-}}$deficient MEFs, and between HLFs from patients with and without IPF.

Results Cyclical stretch induced a significant increase in pSmad2 expression after 48 hours in both MEFs and HLFs $(p<0.05)$. However, stretch did not affect total $\alpha$ SMA protein expression in either cell type. There was no 'dose-response' relationship in stretch-induced pSmad2 expression in WT MEFs (5\%, 10\%, 15\% and 20\% elongation). MEFs deficient in $G_{\alpha q / 11}$ signalling, but not $G_{\alpha 12 / 13}$ signalling, activated significantly less TGF $\beta$ than WT MEFs in response to cyclical stretch $(p<0.05)$. HLFs from both IPF and non-IPF donors activated TGF $\beta$ after 48 hours of cyclical stretch, but IPF fibroblasts activated significantly more TGF $\beta$ than fibroblasts from patients without IPF (6 IPF and 6 non-IPF cell lines; $\mathrm{p}<0.05)$.

Conclusion Cyclical stretch is a physiologically relevant stimulus that drives $G_{\alpha q / 11}$-mediated TGF $\beta$ activation in lung fibroblasts. IPF fibroblasts have enhanced stretch-mediated TGF $\beta$ activation, indicating that dysregulation of breathing-related stretch signalling is a potential mechanism of IPF progression. A greater understanding of these pathways may identify targets for new therapies that could halt the progression of IPF. 